\title{
脱镁叶绿酸的外接环转换与芳环取代叶绿素降解衍生物的合成
}

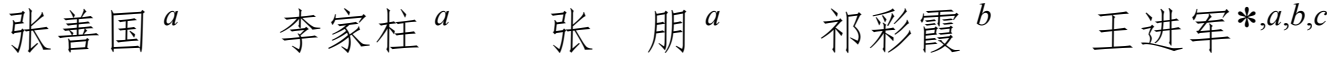 \\ ( ${ }^{a}$ 烟台大学化学化工学院 烟台 264005)

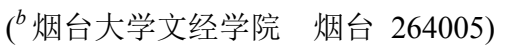 \\ ( ${ }^{c}$ 山东省黄金工程技术研究中心(工业应用) 烟台 264005)
}

\begin{abstract}
摘要 从脱镁叶绿酸-a 甲酯开始, 先行通过 1,3-偶极环加成、游离基取代和 Wittig 成烯等经典反应、在二氢卟吩周环 的 3-, 12-和 20-位上构建了能与色基形成不同共轭效应的含芳取代基团, 再利用空气氧化、缩合和重排反应实现外接环 的结构转化而形成其他各种叶绿素降解结构, 完成了一系列未见报道的新型二氢卟吩衍生物的合成, 其化学结构均经 $\mathrm{UV}, \mathrm{IR},{ }^{1} \mathrm{H}$ NMR 及元素分析予以证实, 同时, 讨论了周环的化学反应历程、含芳取代基团的共轭效应和大环分子电子 光谱的变化规律.
\end{abstract}

关键词 叶绿素-a; 二氢卟吩; 外接环转换; 叶绿素降解产物; 化学反应

\section{Transformation for Exocyclic Ring of Pheophorbide and Syn- thesis of Chlorophyllous Degradation Derivatives}

\author{
Zhang, Shanguo $^{a} \quad$ Li, Jiazhu $^{a} \quad$ Zhang, Peng $^{a} \quad$ Qi, Caixia $^{b} \quad$ Wang, Jinjun ${ }^{* a, b}$ \\ ( ${ }^{a}$ College of Chemistry and Chemical Engineering, Yantai University, Yantai 264005) \\ ( ${ }^{b}$ Wenjing College, Yantai University, Yantai 264005) \\ ( ${ }^{c}$ Shandong Applied Research Centre of Gold Nanotechnology (Au-SDARC), Yantai 264005)
}

\begin{abstract}
From pheophorbide-a methyl ester, the substituted groups with aromatic ring, which can conjugated with chromophore in different forms, were established at 3-, 12- and 20-position on the chlorin periphery by some classic reactions such as 1,3-dipolar cycloaddition, radical substitution and Wittig olefin-forming. The transformations in the exocyclic ring to other chlorophyllous degradation structures were carried out making use of allomerization, condensation and rearrangement reactions. A series of unreported new chlorins were synthesized and their chemical structures were characterized by elemental analysis, UV, IR and ${ }^{1} \mathrm{H}$ NMR spectra. In the same time the chemical reaction process around the periphery, the conjugated effect about the aromatic substitutes and the varying law about electronic spectra of the macrocyclic molecule were also discussed.
\end{abstract}

Keywords chlorophyll-a; chlorin; transformation for exocyclic ring; chlorophyllous degradation derivative; chemical reaction

叶绿素-a 的五元外接环是其二氢卟吩色基所连带 的标识性化学结构, 强直的刚性架构和活泼的 $\beta$-酮酯官 能基团在一定程度上决定着叶绿素的各种属性 ${ }^{[1]}$. 在叶 绿素化学的研究中, 外接环的转化可以衍生出不同的叶 绿素降解产物, 并且能够起到衔接和扩展二氢卟吩大环 碳络的特殊作用, 也是获取具有叶绿素基本碳架的二氢 卟吩衍生物的重要合成策略 ${ }^{[2,3]}$. 叶绿素衍生物的电子
光谱和其他光学属性是表征其应用价值的主要参数, 而 修饰外接环结构和引进能与色基形成各种共轭效应的 取代基团都可以有效地改善大环分子的光物理性质. 为 了深化和拓展叶绿素化学的基础研究, 我们基于前期的 工作基础 ${ }^{[\sim 7]}$, 设计在周环上先行引入含有芳环结构的 取代基团, 再通过外接环的结构转换, 合成出了一系列 未见报道的新型叶绿素降解衍生物.

\section{*E-mail: wjj1955@163.com}

Received August 9, 2014; revised October 23, 2014; published online January 6, 2015.

Project supported by the National Natural Science Foundations of China (No. 21272048) and the Project of Shandong Applied Reaearch Centre of Gold Nanotechnology (2011).

国家自然科学基金(No. 21272048)和山东省黄金工程技术研究中心(2011 年度)资助项目. 
以脱镁叶绿酸-a 甲酯 $(\mathbf{1}$, 简称 $\mathrm{MPa})$ 为起始原料, 与 吡啶-2-甲醛肜经 $N$-溴代琥珀酰亚胺(NBS)溴代和胺催 化脱溴化氢所得的氧化苯甲腈发生 1,3 -偶极环加成反 应, 分离出 $65 \%$ 的 3-吡啶异啞唑啉取代的 MPa 2, 与起 始原料一样 ${ }^{[7]}$, 继续在乙酸中回流, 也能够脱去 $13^{2}$-位 甲氧甲酰基, 以 $64 \%$ 的产率降解为焦脱镁叶绿酸同系物 3; 改在碱性条件下进行空气氧化, 二氢卟吩 2 则转化成 $\mathrm{C}(3)$-位上具有相同取代结构的红紫素-18 4 (43\%). MPa (1)在吡啶中的长时间回流, 同样可以完成外接环中 $\beta$ 酮酯的结构转换 ${ }^{[7]}$, 所得焦脱镁叶绿酸-a 甲酯 5 (简称 $\mathrm{MPPa})$ 与过氧化苯甲酰在甲苯中 $90{ }^{\circ} \mathrm{C}$ 搅拌, 顺利地在 20-meso 位上发生游离基取代反应, 并给出 40\%的 20苯甲酰氧基 MPPa 6. 根据叶绿素能够与空气发生氧化 反应的特性 ${ }^{[4 \mathrm{a}]}$, 在氢氧化锂促进下, 外接环酮与单线态 氧的化学反应在 $13^{2}$-位上形成了另一羰基, 以 $39 \%$ 的收 率生成 20-位取代的二氢卟吩二酮 7 (Scheme 1).

在室温条件下，12-甲酰基焦脱镁叶绿酸-a 甲酯(8) 在氢氧化钠醇水溶液中与茮基三苯基溴化鏻作用, 选择 性地得到具有 $E$ 式结构的 MPPa 9 (67\%), 在 12-位上顺 利地引进了苯甲叉基; 经过与制备化合物 7 相同的碱催 化空气氧化, 其外接环同样能够转化成五元环戊二酮结
构，以 $50 \%$ 的产率形成二氢卟吩二酮 $\mathbf{1 0}$, 在碱性条件下 继续实施空气氧化 ${ }^{[4 \mathrm{a}]}$, 则得到 46\%的 12-苯甲叉基取代 的红紫素-18 11, 在氢氧化钾醇溶液中, 其六元外接环 酐开裂成二酯, 分离出 $58 \%$ 的二氢卟吩 $-e_{6}$ 衍生物 12 ; 选 择盐酸差胺对其环酐实施胺解, 高产率地得到 $N$-取代 红紫素-18 酰亚胺 13. 邻苯二胺与二氢卟吩二酮 10 在含 有三氟乙酸的吡啶溶液中的合环反应，除了得到作为正 常缩合产物的喹喔啉并焦脱镁叶绿酸-a 甲酯(14，18\%) 以外, 还分离出在外接环上发生氧化重排的苯并咪唑并 红紫素-18 15 (16\%) (Scheme 2) ${ }^{[7]}$.

\section{1 结果与讨论}

\section{1 二氢卟吩周环上含芳基团的引入及其产物的结构} 表征

氧化苯甲腈的三原子四电子 $\pi$-体系具有类似于烯 丙基负离子的分子轨道, 作为亲偶极体的 C(3)-乙烯基 与其所发生的 1,3-偶极环加成反应形成了良好的区域选 择性，在 3-位上构筑了具有 $R / S$ 构型的吡啶基取代的二 氢异噁唑基结构. 化合物 2 在 $\delta 7.16$ 处的三重单氢吸收 峰清晰地反映出二氢异噁唑基的氧原子应该连接在

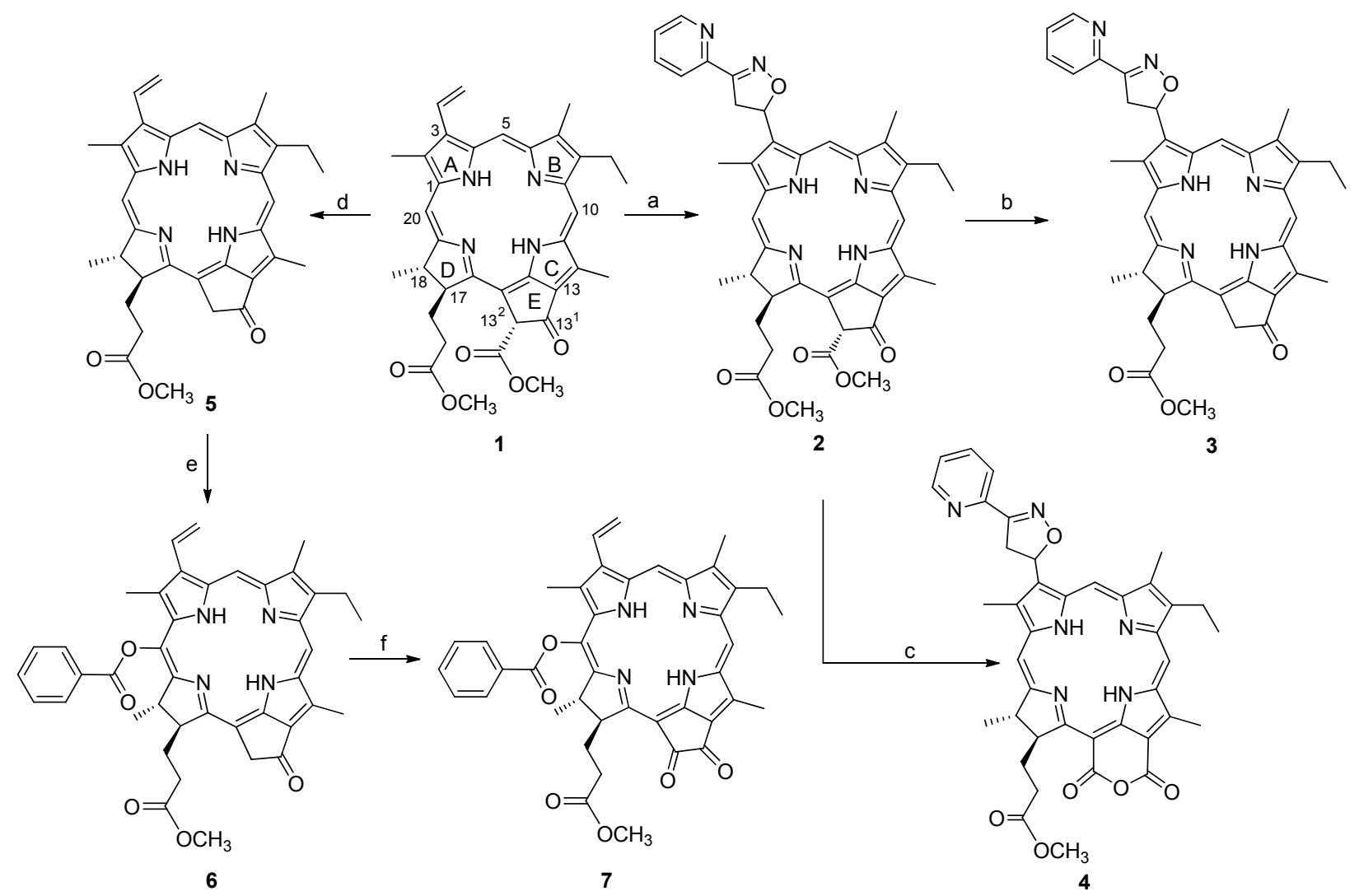

Reagent and conditions: (a) $\mathrm{C}_{5} \mathrm{H}_{4} \mathrm{NCHNOH} / \mathrm{NBS} / \mathrm{TEA}$; (b) $\mathrm{AcOH} / 110{ }^{\circ} \mathrm{C}$; (c) $\mathrm{KOH} / \mathrm{MeOH} / \mathrm{H}_{2} \mathrm{O}$; (d) Pyr/reflux; (e) $(\mathrm{PhCO})_{2} \mathrm{O} / \mathrm{PhCH}{ }_{3}$; (f) $\mathrm{LiOH} /$ $\mathrm{THF} / \mathrm{MeOH}$.

图式 1 叶绿素降解产物在 3-位和 20-位的芳环引入

Scheme 1 Aromatic ring introductions of chlorophyllous degradation products at 3- and 20-position 


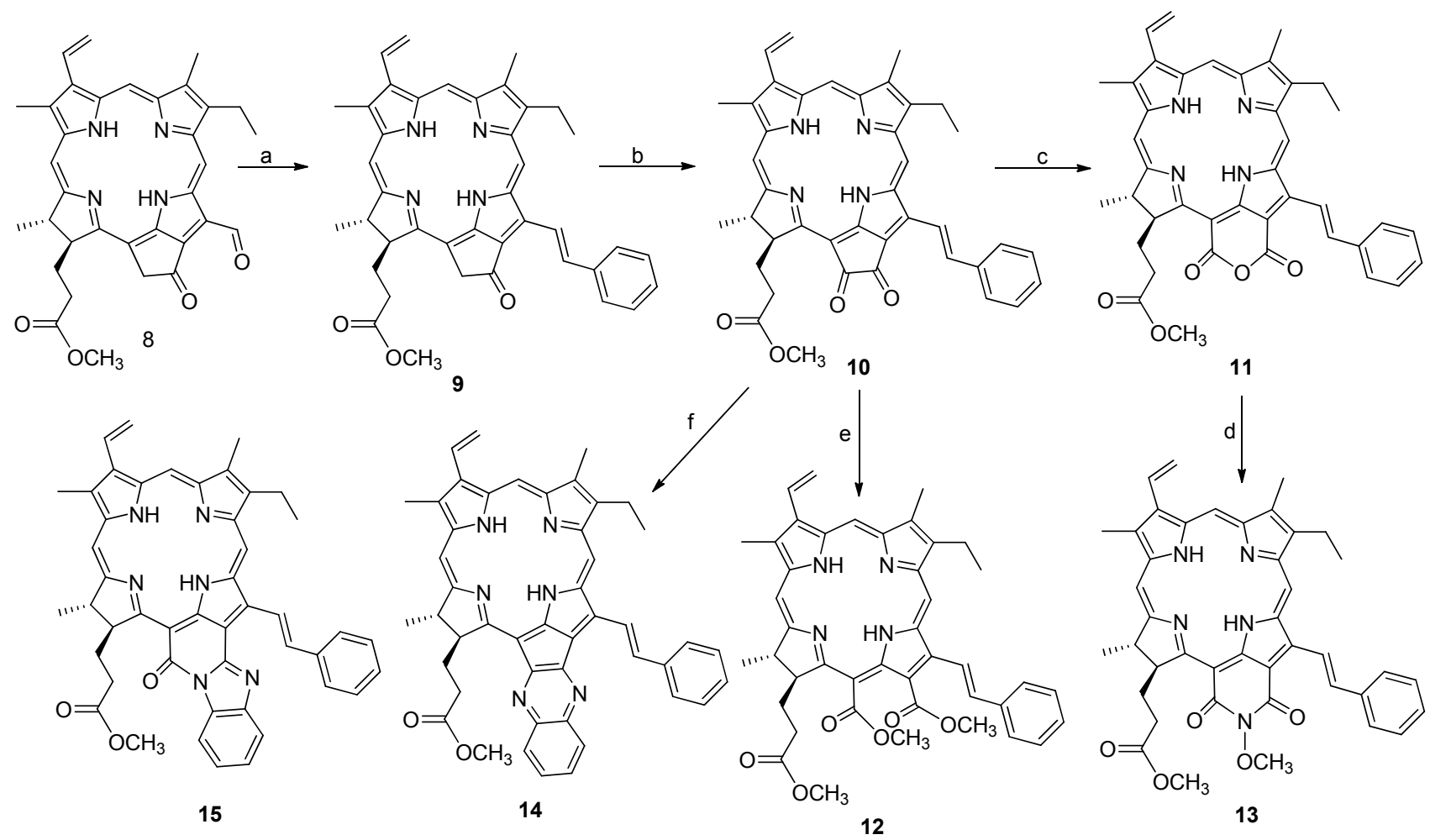

Reagent and conditions: (a) $\mathrm{Ph}_{3} \mathrm{PCH}_{2} \mathrm{Br} / \mathrm{NaOH}$; (b) $\mathrm{LiOH} / \mathrm{THF} / \mathrm{MeOH}$; (c) $\mathrm{KOH} / \mathrm{NeOH} / \mathrm{TEA}$; (d) $\mathrm{Pyr} / \mathrm{NH}_{2} \mathrm{OH}$; (e) $\mathrm{NaOMe} / \mathrm{MeOH}$; (f) $\mathrm{Ph}\left(\mathrm{NH}_{2}\right)_{2} / \mathrm{Pyr} / \mathrm{TFA}$.

图式 2 二氢卟吩的外接环转换与 12-位芳环的引入

Scheme 2 Exocyclic ring transformation and aromatic ring introduction at 12-position for chlorins

乙烯基的 $3 \mathrm{a}$ 位上, 如果氧原子与 $3 \mathrm{~b}$ 位上的碳原子形成 连接, 3a-位上氢质子的振动频率将大幅度地高场移动. 另外, 氢谱中所出现的某些成对吸收信号则确定其加成 产物是由一对差向异构体所组成, 也间接地标示出二氢 卟吩与氧化苯甲腈的反应是从大环色基的上下两面进 行环合. 化合物 8 的 12-位甲酰基与苄基三苯基溴化鏻 的 Wittig 反应与其他位置上的类似反应不同 ${ }^{[7]}$, 结果没 有形成两个 $E$-和 $Z$-式的烯键结构, 而是给出单一的 $E$ 式产物, 其双键上两个单氢的偶合常数为 $15.8 \mathrm{~Hz}$, 所 以, 12a-和 $12 \mathrm{~b}$-位置上的两个质子应该处于双键的两侧, 由于靠近大环色基的 12a-位质子受到氮杂轮烯环电流 强烈的去屏蔽作用, 其吸收信号大幅低场移动至 $\delta 8.46$ 处, 振动频率甚至与 20-meso-位氢的强度基本相同.

20-位的电荷密度高于其他两个 meso-位是多氮杂 轮烯芳香体系的一个独特属性, 最明显的例证来自于叶 绿降解产物的亲电取代反应, 与多种亲电试剂的所有反 应几乎全部发生在 20-meso-位上 ${ }^{[1 \mathrm{cc}]}$. 与亲电取代反应相 似, 游离基反应也经常选择发生在多电子的反应区域, 虽然电子效应对反应的影响程度不如游离基型亲电取 代反应那么敏感, 但过氧化苯甲酰与 MPPa 的取代反应 却全部选择发生在 20-meso-位上, 一方面验证了大环色
基的电子效应对取代反应所形成的影响，同时也验证了 20-位的周边立体环境在取代反应中比其他 meso-位更 为有利. 过氧化苯甲酰在加热条件下裂分为两个苯甲酰 氧游离基, 其一进攻 MPPa 5 形成游离基中间体 $\mathbf{A}$ (图 1), 另一苯甲酰氧游离基夺取 20-位氢原子并形成 20-苯甲 酰氧基取代的焦脱镁叶绿酸-a 甲酯 6 . 与 MPPa 的核磁 共振氢谱相比，化合物 $\mathbf{6}$ 的 20-meso-位质子的单氢吸收 信号已经消失, 在 $\delta 7.76 \sim 8.63$ 范围内所出现的 5 氢三 簇多重峰则清晰地显示出 20 -位上连有一个单取代的苯 基, 而处于低场的 $\delta 8.63$ 位置上裂分为双重峰的双氢信 号则进一步证实芳环上连有能够形成去屏蔽作用的强 吸电子性的酰基.

\section{2 二氢卟吩外接环的结构转换与其电子光谱的变化}

叶绿素类二氢卟吩的电子光谱是衡量是否具有实 际应用意义的最为重要的表征数据, 其色基中的 $\mathrm{N}^{21}-\mathrm{N}^{23}$ 轴向的取代基团对大环分子的紫外-可见光的吸 收区间起到决定性的作用，特别是处于 C-D 环端向的外 接环的结构可以强烈地影响电子光谱中的最大可见光 的吸收波长(Qy 吸收). 根据周环上芳基的结构特点, 对 大环分子的紫外-可见光光谱能够形成不同的电子效 


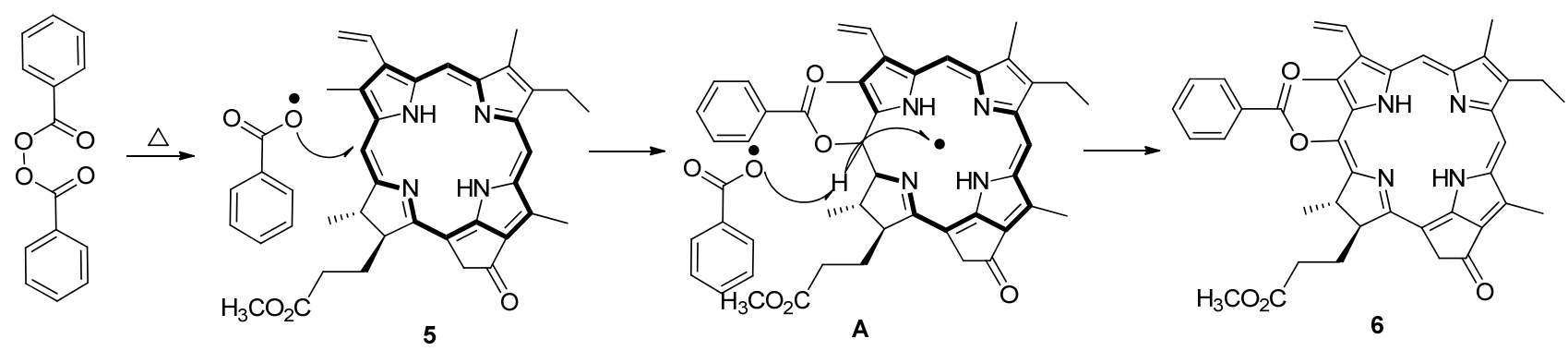

图 1 焦脱镁叶绿酸-a 甲酯与过氧化苯甲酰的游离基取代反应

Figure 1 The radical substitution of methyl pyropheophorbide-a with benzoy peroxide

应, 引进结构的位置、几何形状和空间构型均对其诸多 应用方面产生特定的影响 ${ }^{[8]}$. MPPa 在 12-位上引入苯甲 叉基以后, 大环的共轭体系得以明显的延伸, 其最大可 见光的吸收信号也出现 $12 \mathrm{~nm}$ 的红移(MPPa 的 $\mathrm{Qy}$ 为 668 $\mathrm{nm}$ ); 异噁唑啉基与 3-位乙烯基的交换, 由于缺失了环 外碳碳双键, 取而代之的碳氧单键和碳碳单键都不能完 成环外共轭体系与芳香性色基连通, 因而导致其 Qy 吸 收波长形成小幅蓝移 $(\Delta \mathrm{Qy}=3 \mathrm{~nm}) ; 20$-位苯甲酰氧基的 形成稍微提高了分子的最大可见光的吸收, 虽然苯甲酰 氧基平面与大环平面处于相互垂直的构象, 但与邻近的 2-位和 19-位甲基还是形成很强的空间排斥, 实际上对 大环的平面起到一个伸拉的作用, 改善了大环 $\pi$-体系的 共轭效果, 最终导致 $2 \mathrm{~nm}$ 的微弱红移. 当周环上含芳结 构确定以后, 其外接环的改变与分子的最大可见光的吸 收形成了密切的关联, 脱镁叶绿酸的五元外接环上的 $13^{2}$-上连有一个与 17 -位尾端酯基相反取向的一个甲氧 甲酰基, 由于它的空间排斥作用进一步加大了 $13^{1}$-位酮 羰基对大环平面的偏移, 因此, 脱掉 $13^{2}$-位的酯基以后, 其最大可见光的吸收谱红移了 $2 \mathrm{~nm} .13^{2}$-位上两个偕质 子的化学位移存在着明显的差别, 反映出 MPPa 的外接 环羰基与大环平面并不是完全平行的, 通过五元环上的 酮羰基对邻近质子所施加的磁各向异性效应可以判定 其指向趋于 17 -位尾端酯基的平行方向 ${ }^{[9]}$. 当外接 E-环 中的亚甲基转化成宸基的时候, $13^{2}$-位碳原子的杂化形 式也由 $\mathrm{sp}^{3}$ 变为 $\mathrm{sp}^{2}$, 由于环上五个碳原子都是 $\mathrm{sp}^{2}$ 杂化, 所形成角张力的拉直效应, 在一定程度上促进了外接环 与大环色基共平面, 再加上 $13^{2}$-位上新建立羰基的吸电 子性共轭作用, 使得大环分子的最大可见光的吸收波长 红移至 $708 \mathrm{~nm}(\mathbf{9} \rightarrow \mathbf{1 0})$. 化合物 $\mathbf{1 0}$ 在碱性空气氧化中所 转化的两个产物的最大可见光吸收存在着很大的差异, 二氢卟吩 $-e_{6} 12$ 的 Qy 吸收要比红紫素-18 11 的相应波长 要短 $43 \mathrm{~nm}$, 甚至比 10 的最大可见光的吸收波长还要蓝 移 $27 \mathrm{~nm}$; 虽然三者在 13-和 15-位上都连有羰基, 但 Qy 的吸收位置却相差甚远, 相比之下, 缺少外接环的二氢 卟吩 12 的最大可见光的吸收能量最大. 由于缺乏外接 环的牵固作用, 13-和 15-位上的酯炭基可以绕着所连的
键轴自由旋转, 两个 $\mathrm{C}=\mathrm{O}$ 双键不能始终与大环保持着 $\pi-\pi$ 共轭，因而影响了二氢卟吩色基的电子离域体系向 13-和 15-位取代基团进行进一步的扩展 $(10 \rightarrow \mathbf{1 2})$; 红紫 素-18 的分子碳架则不同, 其外接环中的两个羰基固定 在相应的位置上而与大环 $\pi$-体系形成特定的交盖, 与二 氢卟吩二酮 9 的外接环相比, 相当于邻位二酮中间多嵌 入一个氧原子, 所以缓解了外接环的角张力和键刚性, 缩小了环上羰基 $\pi$-键与大环平面所形成的偏移，从而降 低了大环分子 $\pi$-体系中的最高占有轨道和最低空轨道 的能级差别, 使其 Qy 吸收谱带出现在 $724 \mathrm{~nm}$ 处 $(10 \rightarrow$ 11). 二氢卟吩 11 继续与盐酸差胺的胺解反应形成了新 的二酰亚胺外接环结构, 并促成 Qy 吸收进一步发生红 移. 红紫素-18 与红紫素-18 二酰亚胺中的六元外接环中 夹杂的杂原子不同, 也是造成二者最大可见光吸收形成 $6 \mathrm{~nm}$ 差异的主要原因 $(\mathbf{1 1} \rightarrow \mathbf{1 3})$, 外接环上的杂原子(无 论氧原子还是氮原子)都是以 $\mathrm{sp}^{3}$ 的形式杂化, 氧原子中 两个 $\mathrm{sp}^{3}$ 轨道中的一个可以部分地与邻位羰基的 $\pi$-键交 盖, 进而可以参与大环的离域体系; 与氧原子相比, 氮 原子对非键电子的控制能力较差, 加上与甲氧基相连的 $\mathrm{sp}^{3}$ 轨道又占据着较大的空间, 对另一非键的 $\mathrm{sp}^{3}$ 轨道形 成较大的排斥力量, 使其与邻位羰基的 $\pi$-键进一步趋于 平行, 所以氮原子的非键 $\mathrm{sp}^{3}$ 轨道参与共轭的能力更强 (图 2 中的 A 框).

二氢卟吩二酮 9 的外接环中的邻位二酮与邻苯二胺 的缩合反应给出两个主要产物 14 和 $\mathbf{1 5}$, 与其前体相比, 其最大可见光的吸收波长分别大幅度红移了 30 和 42 $\mathrm{nm}$. 无论是喹喔啉并合焦脱镁叶绿酸的五元外接环还 是苯并咪唑外接红紫素-18 的六元环䣶, 都大面积地扩 展了二氢卟吩大环的共轭体系, 同时, 由于含苯并氮杂 环并入所形成的拉伸作用也有效地提高了四吡咯分子 $\pi$-体系的共平面, 因此其 Qy 吸收谱带形成强烈的红移.

\section{2 结论}

通过周环上的 1,3-偶极环加成、游离基取代、Wittig 成烯和空气氧化等经典的化学反应, 先行在 $\mathrm{N}^{21}-\mathrm{N}^{23}$ 轴 向和 20-meso-位上建立多种含芳基团, 利用外接环的进 


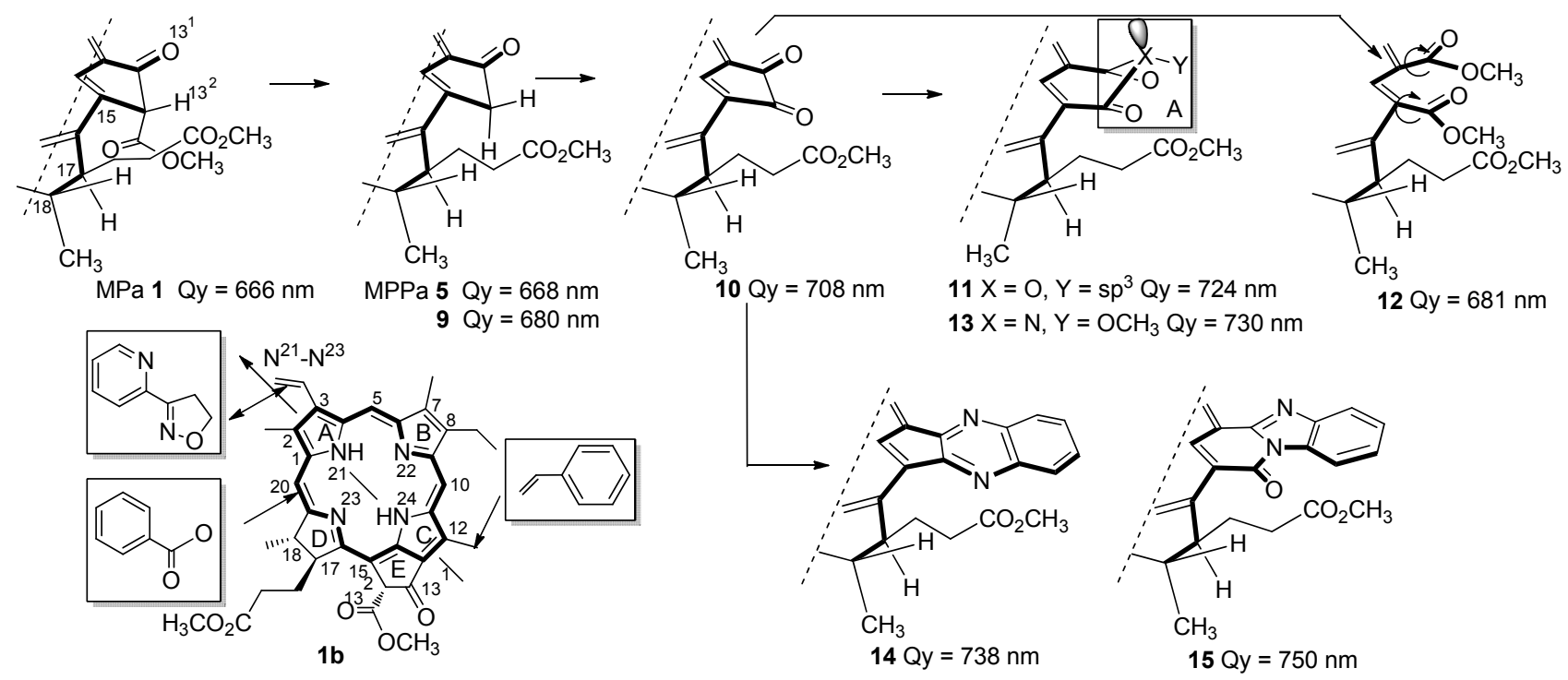

图 2 叶绿素类二氢卟吩外接环的转换与其最大可见光的吸收波长

Figure 2 Transformation for exocyclic ring of pheophorbide and their Qy peak mximums

一步转化形成了其他叶绿素的降解结构; 周环上所构建 的能与色基形成不同共轭效应的含芳取代基团, 可以改 善二氢卟吩中芳香性氮杂轮烯的 $\pi$-电子体系, 而外接环 的结构变化则对大环分子的电子光谱产生明显的影响, 并在一定程度上可以改变了大环分子的化学反应活性. 因此, 利用连有特定结构的叶绿素降解产物的外接环转 换, 是获取新型叶绿素类二氢卟吩衍生物的有效方法.

\section{3 实验部分}

\section{1 仪器与试剂}

元素分析用 Perkin-Elmer 2400 型元素分析仪测定; IR 用 Perkin-Elmer 1730 型红外分光光度仪测定 $(\mathrm{KBr}$ 压 片); UV-Vis 用 UV-160A 型紫外分光光度计测定; ${ }^{1} \mathrm{H}$ NMR 用 Brucker ARX-400 型核磁共振仪测定, 内标为 TMS. 所用试剂均为分析纯或化学纯, 以常法干燥; 焦 脱镁叶绿酸-a 甲酯(1)和焦脱镁叶绿酸-a 甲酯(5)分别按 文献 $[7,10]$ 制备; 12-苯亚甲基焦脱镁叶绿酸-a (8) 按文献 [9]制备.

\section{$3.23-\left[5^{\prime}-(R, S)-\left(3^{\prime}-\left(2^{\prime \prime}-\right.\right.\right.$ 吡啶 基 )-4', $5^{\prime}-$ 二氢异噁唑 基)]-3-去乙烯基脱镁叶绿酸-a 甲酯(2)的合成}

将 $107 \mathrm{mg}(0.464 \mathrm{mmol}) \mathrm{NBS}$ 悬浮于 $2 \mathrm{~mL}$ 干燥二氯 甲烷中, 一次性加入 $105 \mathrm{mg}$ 2-吡啶酫肜, $20 \mathrm{~min}$ 后溴化 结束, 再加入 $89 \mathrm{mg} \mathrm{MPa}(\mathbf{1}, 0.147 \mathrm{mmol})$, 待其完全溶 解后, 将溶解在 $2 \mathrm{~mL}$ 二氯甲烷中的 $102 \mathrm{mg}$ 三乙胺于 15 $\min$ 内滴入反应液, 氮气保护, 室温搅拌反应 $12 \mathrm{~h}$ 后加 入 $15 \mathrm{~mL}$ 二氯甲烷和 $25 \mathrm{~mL}$ 水, 水层用二氯甲烷萃取 $(15 \mathrm{~mL} \times 3)$, 合并有机相并水洗, 用无水硫酸钠干燥后 减压浓缩, 剩余物经柱层析分离[洗脱剂: $V$ 乙酸酷：
$\left.V_{\text {正已㟋 }}=1: 3\right]$, 得 $69 \mathrm{mg}$ 墨绿色固体 $2(0.095 \mathrm{mmol})$, 产 率 65\%. m.p. $236 \sim 239{ }^{\circ} \mathrm{C}$; UV-vis $\left(\mathrm{CH}_{2} \mathrm{Cl}_{2}\right) \lambda_{\max }$

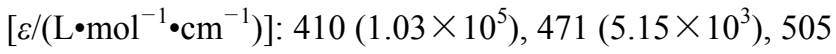
$\left(1.03 \times 10^{4}\right), \quad 536 \quad\left(9.27 \times 10^{3}\right), \quad 608 \quad\left(8.24 \times 10^{3}\right), \quad 666$ $\left(5.46 \times 10^{4}\right) \mathrm{nm} ;{ }^{1} \mathrm{H}$ NMR $\left(\mathrm{CDCl}_{3}\right) \delta:-1.76($ br s, $1 \mathrm{H}$, $\mathrm{NH}), 0.36$ (br s, $1 \mathrm{H}, \mathrm{NH}), 1.61$ (t, $J=7.6 \mathrm{~Hz}, 3 \mathrm{H}, 8 \mathrm{a}-\mathrm{CH}_{3}$ ), $1.82\left(\mathrm{~d}, J=7.2 \mathrm{~Hz}, 3 \mathrm{H}, 18-\mathrm{CH}_{3}\right), 2.20 \sim 2.37(\mathrm{~m}, 2 \mathrm{H}$, $17 \mathrm{a}+17 \mathrm{~b}-\mathrm{H}), 2.47 \sim 2.69(\mathrm{~m}, 2 \mathrm{H}, 17 \mathrm{a}+17 \mathrm{~b}-\mathrm{H}), 3.54$ (q, $J=7.6 \mathrm{~Hz}, 3 \mathrm{H}, 8 \mathrm{~b}-\mathrm{H}$ ), 2.97, 3.42, 3.57, 3.66, 3.89 (each s, $\left.3 \mathrm{H}, \mathrm{CH}_{3}+\mathrm{OCH}_{3}\right), 4.17 \sim 4.25\left(\mathrm{~m} 2 \mathrm{H}, 18-\mathrm{H}+4{ }^{\prime}-\mathrm{H}\right)$, $4.42 \sim 4.52\left(\mathrm{~m}, 1 \mathrm{H}, 17-\mathrm{H}+4^{\prime}-\mathrm{H}\right), 6.27\left(\mathrm{~s}, 1 \mathrm{H}, 13^{2}-\mathrm{H}\right), 7.16$ (t, $\left.J=10.9 \mathrm{~Hz}, 1 \mathrm{H}, 5^{\prime}-\mathrm{H}\right), 7.37$ (t, $\left.J=4.8 \mathrm{~Hz}, 1 \mathrm{H}, \mathrm{Py}-\mathrm{H}\right)$, 7.87 (t, $J=7.8 \mathrm{~Hz}, 1 \mathrm{H}, \mathrm{Py}-\mathrm{H}), 8.37$ (d, $J=7.8 \mathrm{~Hz}, 1 \mathrm{H}$, Py-H), 8.64 (d, $J=4.8 \mathrm{~Hz}, 1 \mathrm{H}, \mathrm{Py}-\mathrm{H}), 8.60,9.34$ (9.33), 9.45 (9.44) (each s, each $1 \mathrm{H}$, meso-H); IR (KBr) v: 3476 $(\mathrm{N}-\mathrm{H}), 2927,2854(\mathrm{C}-\mathrm{H}), 1741,1700,1685(\mathrm{C}=\mathrm{O})$, $1633(\mathrm{C}=\mathrm{C}), 1556$ (chlorin skeleton), 1464, 1355, 1261, $1101,806 \mathrm{~cm}^{-1}$. Anal. calcd for $\mathrm{C}_{42} \mathrm{H}_{42} \mathrm{~N}_{6} \mathrm{O}_{6} \mathrm{C} 69.41, \mathrm{H}$ 5.82, N 11.56; found C 69.33, H 5.76, N 11.79.

\section{$3.33-\left[5^{\prime}-(R, S)-\left(3^{\prime}-\left(2^{\prime}-\right.\right.\right.$ 吡啶 基 )-4',5'- 二氢 异噁唑 基)]-3-去乙烯基焦脱镁叶绿酸-a 甲酯(3)的合成}

将 $169 \mathrm{mg} 2(0.233 \mathrm{mmol})$ 溶解于 $30 \mathrm{~mL}$ 乙酸中, $110^{\circ} \mathrm{C}$ 搅拌反应 $4 \mathrm{~h}$, 冷却后加入 $150 \mathrm{~mL}$ 冰水和 $80 \mathrm{~mL}$ 二氯甲烷, 水层用二氯甲烷萃取 $(25 \mathrm{~mL} \times 3)$, 合并有机 相并水洗, 用无水硫酸钠干燥后浓缩, 剩余物经柱层析

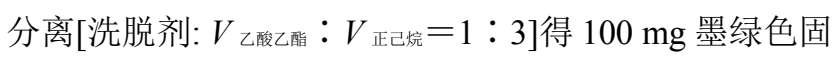
体 3 (0.149 mmol), 产率 64\%. m.p. $228 \sim 230{ }^{\circ} \mathrm{C}$; UV-vis

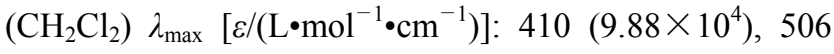


$\left(9.88 \times 10^{3}\right), \quad 536 \quad\left(8.89 \times 10^{2}\right), \quad 608 \quad\left(6.92 \times 10^{3}\right), \quad 666$ $\left(5.04 \times 10^{4}\right) \mathrm{nm} ;{ }^{1} \mathrm{H}$ NMR $\left(\mathrm{CDCl}_{3}\right) \delta:-1.57($ br s, $1 \mathrm{H}$, $\mathrm{NH}), 0.36$ (br s, $1 \mathrm{H}, \mathrm{NH}), 1.61$ (t, $J=7.6 \mathrm{~Hz}, 3 \mathrm{H}, 8 \mathrm{a}-\mathrm{CH}_{3}$ ), $1.82\left(\mathrm{~d}, J=7.2 \mathrm{~Hz}, 3 \mathrm{H}, 18-\mathrm{CH}_{3}\right), 2.20 \sim 2.37(\mathrm{~m}, 2 \mathrm{H}$, $17 \mathrm{a}+17 \mathrm{~b}-\mathrm{H}), \quad 2.47 \sim 2.69$ (m, $2 \mathrm{H}, 17 \mathrm{a}+17 \mathrm{~b}-\mathrm{H}), 3.00$, 3.53, 3.62, 3.83 (each s, $3 \mathrm{H}, \mathrm{CH}_{3}+\mathrm{OCH}_{3}$ ), 3.54 (q, $J=7.6$ $\left.\mathrm{Hz}, 2 \mathrm{H}, 8 \mathrm{a}-\mathrm{CH}_{2}\right), 4.11 \sim 4.25\left(\mathrm{~m}, 2 \mathrm{H}, 18-\mathrm{H}+4^{\prime}-\mathrm{H}\right), 4.42 \sim$ $4.52\left(\mathrm{~m}, 2 \mathrm{H}, 17-\mathrm{H}+4^{\prime}-\mathrm{H}\right), 4.99(\mathrm{~d}, J=20.0 \mathrm{~Hz}, 1 \mathrm{H}$, $\left.13^{2}-\mathrm{H}\right), 5.28\left(\mathrm{~d}, J=20.0 \mathrm{~Hz}, 1 \mathrm{H}, 13^{2}-\mathrm{H}\right), 7.14(\mathrm{t}, J=11.0$ Hz, 1H, 5'-H), 7.34 (t, $J=4.8$ Hz, 1H, Py-H), 7.86 (t, $J=$ $7.8 \mathrm{~Hz}, 1 \mathrm{H}, \mathrm{Py}-\mathrm{H}$ ), 8.37 (d, J=7.8 Hz, 1H, Py-H), 8.61 (d, $J=4.8 \mathrm{~Hz}, 1 \mathrm{H}, \mathrm{Py}-\mathrm{H}$ ), 8.54, 9.29, 9.39 (each s, each $1 \mathrm{H}$, meso-H); IR (KBr) v: $3441(\mathrm{~N}-\mathrm{H}), 2964(\mathrm{C}-\mathrm{H}), 1741$, 1733, $1689(\mathrm{C}=\mathrm{O}), 1654(\mathrm{C}=\mathrm{C}), 1560$ (chlorin skeleton), 1432, 1253, 1169, 1083, $944 \mathrm{~cm}^{-1}$. Anal. calcd for $\mathrm{C}_{40} \mathrm{H}_{40} \mathrm{~N}_{6} \mathrm{O}_{4} \mathrm{C} 71.84, \mathrm{H}$ 6.03, N 12.57; found C 71.90, H 6.19, N 12.69.

\section{$3.43-\left[5^{\prime}-(R, S)-\left(3^{\prime}-\left(2^{\prime \prime}-\right.\right.\right.$ 吡啶 基 )-4',5'- 二氢 异噁唑} 基)]-3-去乙烯基红紫素-18 甲酯(4)的合成

将 $98 \mathrm{mg}$ 化合物 $3(0.147 \mathrm{mmol})$ 溶解于 $30 \mathrm{~mL}$ 氢氧 化钾饱和甲醇溶液中, 室温开口搅拌 $5 \mathrm{~h}$, 滴加乙酸至 $\mathrm{pH}$ 约为 $2 \sim 3$, 加入 $40 \mathrm{~mL}$ 水后, 用二氯甲烷萃取 (15 $\mathrm{mL} \times 3)$, 合并有机层并水洗, 用无水硫酸钠干燥, 减压 除尽溶剂, 将剩余物再溶解于 $15 \mathrm{~mL}$ 乙酸中, 室温搅拌 反应 $12 \mathrm{~h}$, 减压除尽溶剂, 剩余物用 $10 \mathrm{~mL}$ 二氯甲烷溶 解并用重氮甲烷甲基化, 浓缩后用硅胶柱层析分离 [洗

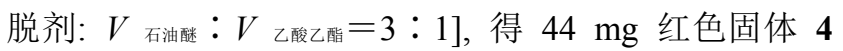
(0.063 mmol), 产率 43\%. m.p. 205 207 ${ }^{\circ} \mathrm{C}$; UV-vis

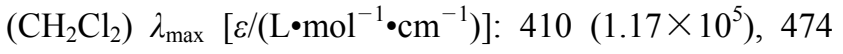
$\left(3.51 \times 10^{3}\right), \quad 504 \quad\left(5.85 \times 10^{3}\right), \quad 542 \quad\left(1.76 \times 10^{4}\right), \quad 638$ $\left(7.02 \times 10^{3}\right), 698\left(3.86 \times 10^{4}\right) \mathrm{nm} ;{ }^{1} \mathrm{H}$ NMR $\left(\mathrm{CDCl}_{3}\right) \delta$ : -0.02 (br s, $1 \mathrm{H}, \mathrm{NH}), 0.23(\mathrm{br} \mathrm{s}, 1 \mathrm{H}, \mathrm{NH}),-1.61$ (t, $J=$ $\left.7.6 \mathrm{~Hz}, 3 \mathrm{H}, 8 \mathrm{~b}-\mathrm{CH}_{3}\right), 1.75$ (d, $\left.J=7.2 \mathrm{~Hz}, 3 \mathrm{H}, 18-\mathrm{CH}_{3}\right)$, $1.94 \sim 2.03(\mathrm{~m}, 1 \mathrm{H}, 17 \mathrm{a}+17 \mathrm{~b}-\mathrm{H}), 2.42 \sim 2.48(\mathrm{~m}, 2 \mathrm{H}$, $17 \mathrm{a}+17 \mathrm{~b}-\mathrm{H}), 2.73 \sim 2.78(\mathrm{~m}, 1 \mathrm{H}, 17 \mathrm{a}+17 \mathrm{~b}-\mathrm{H}), 3.55(\mathrm{q}$, $J=6.9 \mathrm{~Hz}, 2 \mathrm{H}, 8 \mathrm{a}-\mathrm{H}$ ), 2.98, 3.40, 3.37, 3.60 (each s, each $\left.3 \mathrm{H}, \mathrm{OCH}_{3}+\mathrm{CH}_{3}\right), 4.28 \sim 4.32\left(\mathrm{~m}, 2 \mathrm{H}, 4^{\prime}-\mathrm{H}\right), 4.41$ (q, $J=$ $7.2 \mathrm{~Hz}, 1 \mathrm{H}, 18-\mathrm{H}), 5.20(\mathrm{~d}, J=8.3 \mathrm{~Hz}, 1 \mathrm{H}, 17-\mathrm{H}), 7.04 \sim$ $7.10(\mathrm{~m}, 1 \mathrm{H}, 5 \mathrm{\prime}-\mathrm{H})$ ), $7.41 \sim 7.90(\mathrm{~m}, 2 \mathrm{H}, \mathrm{Py}-\mathrm{H}), 8.37 \sim$ 8.66 (m, 2H, Py-H), 8.61, 9.41, 9.54 (each s, each 1H, meso-H); IR (KBr) v: $3449 \quad(\mathrm{~N}-\mathrm{H}), 2928(\mathrm{C}-\mathrm{H}), 1740$, $1701(\mathrm{C}=\mathrm{O}), 1654(\mathrm{C}=\mathrm{C}), 1560$ (chlorin skeleton), 1458, 1401, 1073, $670 \mathrm{~cm}^{-1}$. Anal. calcd for $\mathrm{C}_{40} \mathrm{H}_{38} \mathrm{~N}_{6} \mathrm{O}_{6} \mathrm{C}$ 68.75, H 5.48, N 12.03; found C 68.60, H 5.67, N 12.19 .

\subsection{0 -苯甲酰氧基焦脱镁叶绿酸-a 甲酯(6)的合成}

将 $50 \mathrm{mg}$ MPPa $5(0.091 \mathrm{mmol})$ 溶解于 $10 \mathrm{~mL}$ 苯中, 加入 $20 \mathrm{mg}$ 过氧化苯甲酰 $(0.112 \mathrm{mmol}), 90{ }^{\circ} \mathrm{C}$ 搅拌反应 $4 \mathrm{~h}$. 先后加入 $20 \mathrm{~mL}$ 水与 $20 \mathrm{~mL}$ 二氯甲烷，水层用二氯 甲烷萃取 $(25 \mathrm{~mL} \times 3)$, 合并有机相并水洗, 用无水硫酸 钠干燥后减压浓缩, 剩余物经柱层析分离 [洗脱剂: $\left.V_{\text {乙配柾 }}: V_{\text {正已㧤 }}=1: 3\right]$, 得 $24 \mathrm{mg}$ 紫红色固体 $6(0.037$ mmol), 产率 $40 \%$. m.p. $189 \sim 192{ }^{\circ} \mathrm{C}$. UV-vis $\left(\mathrm{CH}_{2} \mathrm{Cl}_{2}\right)$

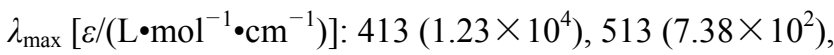
$545\left(1.35 \times 10^{3}\right), 611\left(7.38 \times 10^{2}\right), 670\left(5.17 \times 10^{3}\right) \mathrm{nm} ;{ }^{1} \mathrm{H}$ NMR $\left(\mathrm{CDCl}_{3}\right) \delta$ : -2.20 (br s, $\left.1 \mathrm{H}, \mathrm{NH}\right), 0.45($ br s, $1 \mathrm{H}$, $\mathrm{NH}), 1.72\left(\mathrm{t}, J=7.6 \mathrm{~Hz}, 3 \mathrm{H}, 8-\mathrm{CH}_{3}\right), 1.70(\mathrm{~d}, J=7.1 \mathrm{~Hz}$, $\left.3 \mathrm{H}, 18-\mathrm{CH}_{3}\right), 1.81 \sim 1.90(\mathrm{~m}, 1 \mathrm{H}, 17 \mathrm{a}+17 \mathrm{~b}-\mathrm{H}), 2.27 \sim$ $2.38(\mathrm{~m}, 2 \mathrm{H}, 17 \mathrm{a}+17 \mathrm{~b}-\mathrm{H}), 2.45 \sim 2.57(\mathrm{~m}, 1 \mathrm{H}, 17 \mathrm{a}+$ $17 \mathrm{~b}-\mathrm{H}$ ), 3.73 (q, $J=7.6 \mathrm{~Hz}, 2 \mathrm{H}, 8 \mathrm{a}-\mathrm{H}), 3.27,3.28,3.46$, 3.71 (each s, each $3 \mathrm{H}, \mathrm{OCH}_{3}+\mathrm{CH}_{3}$ ), 4.25 (q, $J=7.3 \mathrm{~Hz}$, $1 \mathrm{H}, 18-\mathrm{H}), 4.28$ (d, $J=8.0 \mathrm{~Hz}, 1 \mathrm{H}, 17-\mathrm{H}), 5.20$ (d, $J=19.9$ $\left.\mathrm{Hz}, 1 \mathrm{H}, 13^{2}-\mathrm{H}\right), 5.31\left(\mathrm{~d}, J=19.9 \mathrm{~Hz}, 1 \mathrm{H}, 13^{2}-\mathrm{H}\right), 6.16(\mathrm{dd}$, $J=17.8,1.5 \mathrm{~Hz}, 1 \mathrm{H}$, trans-3b-H), 6.21 (dd, $J=11.7,1.5$ $\mathrm{Hz}, 1 \mathrm{H}$, cis-3b-H), 7.92 (dd, $J=17.8,11.5 \mathrm{~Hz}, 1 \mathrm{H}, 3 \mathrm{a}-\mathrm{H}$ ), 7.76 (t, $J=7.9 \mathrm{~Hz}, 2 \mathrm{H}, \mathrm{ArH}), 7.87$ (t, $J=7.9 \mathrm{~Hz}, 1 \mathrm{H}$, ArH), 8.63 (dd, $J=8.3,6.2 \mathrm{~Hz}, 2 \mathrm{H}, \mathrm{ArH}$ ), 9.57, 9.58 (each $\mathrm{s}$, each $1 \mathrm{H}$, meso-H); IR $(\mathrm{KBr}) v: 3434(\mathrm{~N}-\mathrm{H}), 2858(\mathrm{C}-$ H), 1739, $1689(\mathrm{C}=\mathrm{O}), 1623(\mathrm{C}=\mathrm{C}), 1562$ (chlorin skeleton), 1460, 1343, 1218, 1160, 1027, $905 \mathrm{~cm}^{-1}$. Anal. calcd for $\mathrm{C}_{41} \mathrm{H}_{40} \mathrm{~N}_{4} \mathrm{O}_{5}$ C 73.63, H 6.03, N 8.38; found $\mathrm{C}$ 73.56, H 6.20, N 8.21.

\subsection{0 -苯甲酰氧基- $13^{2}$-氧代焦脱镁叶绿酸-a 甲酯(7) 的合成}

取 $43 \mathrm{mg}$ 二氢卟吩 $6(0.064 \mathrm{mmol})$ 溶解于 $10 \mathrm{~mL}$ 四 氢呋喃中, 加入 $0.225 \mathrm{~g} \mathrm{LiOH}$ (溶解于 $4 \mathrm{~mL}$ 水中, 用 8 $\mathrm{mL} \mathrm{MeOH}$ 稀释)的开放容器中, 室温避光剧烈摚拌 $6 \mathrm{~h}$, 摚拌下用 $\mathrm{AcOH}$ 调至酸性 $(\mathrm{pH}=3)$, 先后加入 $10 \mathrm{~mL}$ 二 氯甲烷和 $30 \mathrm{~mL}$ 水, 水层用二氯甲烷萃取 $(15 \mathrm{~mL} \times 3)$, 合并有机层, 用无水硫酸钠干燥, 减压蒸干, 再用 $5 \mathrm{~mL}$ 二氯甲烷溶解, 加入过量的重氮甲烷振摇 $2 \mathrm{~min}$ 后, 用 $\mathrm{AcOH}$ 快速淬灭, 再加 $20 \mathrm{~mL}$ 水, 分出有机层, 水层用 二氯甲烷萃取 $(15 \mathrm{~mL} \times 3)$, 合并有机层, 水洗两次, 用 无水硫酸钠干燥, 减压浓缩, 剩余物用硅胶柱层析分离 [洗脱剂: $V$ 甲羑 $: V$ 两曲 $=20: 1$ ], 得 $17 \mathrm{mg}$ 黄色固体 7 (0.025 mmol), 产率 39\%. m.p. 207 209 ${ }^{\circ} \mathrm{C}$; UV-vis

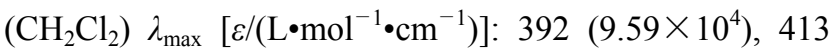
$\left(1.09 \times 10^{5}\right), \quad 515 \quad\left(1.42 \times 10^{4}\right), \quad 628 \quad\left(5.45 \times 10^{3}\right), \quad 680$ $\left(5.12 \times 10^{4}\right) \mathrm{nm} ;{ }^{1} \mathrm{H}$ NMR $\left(\mathrm{CDCl}_{3}\right) \delta:-0.75($ br s, $1 \mathrm{H}$, $\mathrm{NH}),-0.08$ (br s, 1H, NH), 1.68 (1.66) (t, $J=7.6 \mathrm{~Hz}, 3 \mathrm{H}$, 
8- $\left.\mathrm{CH}_{3}\right), 1.72(1.29)\left(\mathrm{d}, J=7.0 \mathrm{~Hz}, 3 \mathrm{H}, 18-\mathrm{CH}_{3}\right), 2.01 \sim$ $2.09(\mathrm{~m}, 1 \mathrm{H}, 17 \mathrm{a}+17 \mathrm{~b}-\mathrm{H}), 2.35 \sim 2.54(\mathrm{~m}, 2 \mathrm{H}, 17 \mathrm{a}+$ 17b-H), $2.73 \sim 2.80$ (m, $1 \mathrm{H}, 17 \mathrm{a}+17 \mathrm{~b}-\mathrm{H}), 3.80$ (3.78), 3.42 (3.60), 3.18 (3.16), 3.14 (each s, each $3 \mathrm{H}, \mathrm{CH}_{3}+$ $\left.\mathrm{OCH}_{3}\right), 3.56$ (3.64) (q, $\left.J=7.6 \mathrm{~Hz}, 2 \mathrm{H}, 8 \mathrm{a}-\mathrm{H}\right), 4.14$ (4.74) (q, $J=7.1 \mathrm{~Hz}, 1 \mathrm{H}, 18-\mathrm{H}), 5.62(5.45)(\mathrm{d}, J=8.2 \mathrm{~Hz}, 1 \mathrm{H}$, 17-H), 6.15 (6.13) (d, $J=17.9 \mathrm{~Hz}, 1 \mathrm{H}$, trans-3b-H), 6.22 (6.21) (d, $J=11.5 \mathrm{~Hz}, 1 \mathrm{H}$, cis-3b-H), 7.52 7.80 (m, 3H, ArH), 7.87 (dd, $J=17.9,11.4 \mathrm{~Hz}, 1 \mathrm{H}, 3 \mathrm{a}-\mathrm{H}), 8.61$ (8.59) (d, $J=7.5 \mathrm{~Hz}, 1 \mathrm{H}, \mathrm{ArH}), 9.64$ (9.59), 9.54 (9.50) (each s, each 1H, meso-H); IR (KBr) v: $3446(\mathrm{~N}-\mathrm{H}), 2956,2927$ $(\mathrm{C}-\mathrm{H}), 1731,1701 \quad(\mathrm{C}=\mathrm{O}), 1623(\mathrm{C}=\mathrm{C}), 1560$ (chlorin skeleton), 1463, 1374, 1232, 1170, 1033, $914 \mathrm{~cm}^{-1}$. Anal. calcd for $\mathrm{C}_{41} \mathrm{H}_{38} \mathrm{~N}_{4} \mathrm{O}_{6}$ C 72.12, H 5.61, N 8.21; found C 72.16, H 5.40, N 8.36;

\subsection{2(E)-12-苯亚甲基焦脱镁叶绿酸-a 甲酯(9)的合 成}

将 $156 \mathrm{mg}$ 二氢卟吩醛 $8(0.277 \mathrm{mmol})$ 溶解于 $10 \mathrm{~mL}$ 二氯甲烷中, 搅拌下加入 $80 \mathrm{mg}$ 溴化茮基三苯基鏻, 再 加入溶解于 $3 \mathrm{~mL}$ 水的 $10 \mathrm{mg}$ 氢氧化钠溶液, 氮气保护, 室温避光搅拌反应 $40 \mathrm{~min}$, 加入 $20 \mathrm{~mL}$ 水, 用二氯甲烷 萃取 $(15 \mathrm{~mL} \times 3)$, 合并有机相, 用无水硫酸钠干燥, 减 压蒸除溶剂, 剩余物经硅胶柱层析 [洗脱剂: $V$ 甲菜: $\left.V_{\text {两醉 }}=10: 1\right]$, 得 $118 \mathrm{mg}$ 黄色产物 $9(0.186 \mathrm{mmol})$, 产率 67\%. m.p. $211 \sim 213{ }^{\circ} \mathrm{C}$; UV-vis $\left(\mathrm{CH}_{2} \mathrm{Cl}_{2}\right) \lambda_{\max }[\varepsilon /(\mathrm{L} \cdot$ $\left.\left.\mathrm{mol}^{-1} \cdot \mathrm{cm}^{-1}\right)\right]: \quad 410\left(1.06 \times 10^{5}\right), 510\left(9.54 \times 10^{3}\right), 542$ $\left(9.54 \times 10^{3}\right), \quad 663 \quad\left(9.12 \times 10^{3}\right), \quad 680 \quad\left(3.92 \times 10^{4}\right) \quad n m ;$ ${ }^{1} \mathrm{HNMR}\left(\mathrm{CDCl}_{3}\right) \delta$ : $-1.09($ br s, $1 \mathrm{H}, \mathrm{NH}), 0.82($ br s, $1 \mathrm{H}$, $\mathrm{NH}), 1.67\left(\mathrm{t}, J=7.6 \mathrm{~Hz}, 3 \mathrm{H}, 8-\mathrm{CH}_{3}\right), 1.81(\mathrm{~d}, J=7.3 \mathrm{~Hz}$, $\left.3 \mathrm{H}, 18-\mathrm{CH}_{3}\right), 2.25 \sim 2.36(\mathrm{~m}, 2 \mathrm{H}, 17 \mathrm{a}+17 \mathrm{~b}-\mathrm{H}), 2.62 \sim$ $2.73(\mathrm{~m}, 1 \mathrm{H}, 17 \mathrm{a}+17 \mathrm{~b}-\mathrm{H}), 2.51 \sim 2.61(\mathrm{~m}, 1 \mathrm{H}, 17 \mathrm{a}+$ $17 \mathrm{~b}-\mathrm{H}), 3.64$ (q, $J=7.6 \mathrm{~Hz}, 2 \mathrm{H}, 8 \mathrm{a}-\mathrm{H}), 3.17,3.34,3.63$ (each s, each $3 \mathrm{H}, \mathrm{OCH}_{3}+\mathrm{CH}_{3}$ ), $4.23(\mathrm{dd}, J=11.8,6.3 \mathrm{~Hz}$, 1H, 17-H), 4.41 (q, $J=7.3 \mathrm{~Hz}, 1 \mathrm{H}, 18-\mathrm{H}), 5.09$ (d, $J=19.7$ $\left.\mathrm{Hz}, 1 \mathrm{H}, 13^{2}-\mathrm{H}\right), 5.24\left(\mathrm{~d}, J=19.7 \mathrm{~Hz}, 1 \mathrm{H}, 13^{2}-\mathrm{H}\right), 6.14(\mathrm{dd}$, $J=11.5,1.0 \mathrm{~Hz}, 1 \mathrm{H}$, cis-3b-H), 6.25 (dd, $J=17.9,1.0 \mathrm{~Hz}$, $1 \mathrm{H}$, trans-3b-H), 7.42 (t, $J=7.3 \mathrm{~Hz}, 1 \mathrm{H}, \mathrm{ArH}), 7.55(\mathrm{t}, J=$ $7.5 \mathrm{~Hz}, 2 \mathrm{H}, \mathrm{ArH}), 7.91$ (dd, $J=17.9,11.5 \mathrm{~Hz}, 1 \mathrm{H}, 3 \mathrm{a}-\mathrm{H}$ ), $7.98(\mathrm{~d}, J=7.5 \mathrm{~Hz}, 2 \mathrm{H}, \mathrm{ArH}), 8.46$ (d, $J=15.8 \mathrm{~Hz}, 1 \mathrm{H}$, 12b-H), 9.10 (d, $J=15.8 \mathrm{~Hz}, 1 \mathrm{H}, 12 \mathrm{a}-\mathrm{H}), 8.41,9.19,9.45$ (each s, each 1H, meso-H); IR (KBr) v: $3505(\mathrm{~N}-\mathrm{H}), 2964$ $(\mathrm{C}-\mathrm{H}), 1731 \sim 1696(\mathrm{C}=\mathrm{O}), 1608(\mathrm{C}=\mathrm{C}), 1545$ (chlorin skeleton), 1409, 1256, 1178, 1070, $907 \mathrm{~cm}^{-1}$. Anal. calcd for $\mathrm{C}_{41} \mathrm{H}_{40} \mathrm{~N}_{4} \mathrm{O}_{3} \mathrm{C} 77.33, \mathrm{H} 6.33, \mathrm{~N} 8.80$; found $\mathrm{C} 77.50, \mathrm{H}$ $6.45, \mathrm{~N} 8.97$.
3.7 12(E)-12-苯亚甲基-13 2 -氧代焦脱镁叶绿酸-a 甲酯 (10)的合成

将化合物 $\mathbf{6}$ 用化合物 9 替代，按照制备化合物 7 的 步骤得黄色固体 10, 产率 50\%; m.p. 224 226 ${ }^{\circ} \mathrm{C}$,

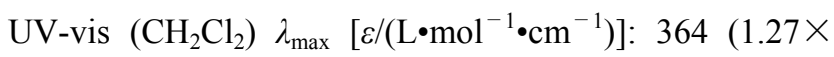
$\left.10^{5}\right), 447\left(1.69 \times 10^{5}\right), 533\left(2.87 \times 10^{4}\right), 648\left(1.86 \times 10^{5}\right)$, $708\left(1.61 \times 10^{5}\right) ;{ }^{1} \mathrm{H}$ NMR $\left(\mathrm{CDCl}_{3}\right) \delta:-2.42($ br s, $1 \mathrm{H}$, $\mathrm{NH}),-0.65($ br s, $1 \mathrm{H}, \mathrm{NH}), 1.30(\mathrm{t}, J=7.6 \mathrm{~Hz}, 3 \mathrm{H}$, $\left.8-\mathrm{CH}_{3}\right), 2.03\left(\mathrm{~d}, J=7.2 \mathrm{~Hz}, 3 \mathrm{H}, 18-\mathrm{CH}_{3}\right), 2.26 \sim 2.38(\mathrm{~m}$, $1 \mathrm{H}, \quad 17 \mathrm{a}+17 \mathrm{~b}-\mathrm{H}), 2.47 \sim 2.54(\mathrm{~m}, 1 \mathrm{H}, 17 \mathrm{a}+17 \mathrm{~b}-\mathrm{H})$, $2.77 \sim 3.05(\mathrm{~m}, 2 \mathrm{H}, 17 \mathrm{a}+17 \mathrm{~b}-\mathrm{H}), 3.00,3.19,3.67$ (each s, each $\left.3 \mathrm{H}, \mathrm{CH}_{3}+\mathrm{OCH}_{3}\right), 3.12(\mathrm{q}, J=7.6 \mathrm{~Hz}, 2 \mathrm{H}, 8 \mathrm{a}-\mathrm{H})$, $4.62(\mathrm{q}, J=7.2 \mathrm{~Hz}, 1 \mathrm{H}, 18-\mathrm{H}), 5.11(\mathrm{~d}, J=8.8 \mathrm{~Hz}, 1 \mathrm{H}$, 17-H), 6.04 (d, $J=11.6 \mathrm{~Hz}$, cis-3b-H), 6.11 (d, $J=17.6$ $\mathrm{Hz}$, trans-3b-H), 6.85 (d, $J=16.0 \mathrm{~Hz}, 1 \mathrm{H}, 12 \mathrm{~b}-\mathrm{H}), 7.38 \sim$ 7.57 (m, 5H, PhH), 7.66 (dd, $J=17.6,11.6 \mathrm{~Hz}, 1 \mathrm{H}, 3 \mathrm{a}-\mathrm{H})$, 8.15 (d, $J=16.0 \mathrm{~Hz}, 1 \mathrm{H}, 12 \mathrm{a}-\mathrm{H}$ ), 8.09, 8.73, 9.20 (each s, each 1H, meso-H); IR (KBr) v: $3454(\mathrm{~N}-\mathrm{H}), 2925(\mathrm{C}-$ $\mathrm{H}), 1730,1708(\mathrm{C}=\mathrm{O}), 1618(\mathrm{C}=\mathrm{C}), 1461$ (chlorin skeleton), 1448, 1380, 1253, 1172, 1083, 1010, 800, $729 \mathrm{~cm}^{-1}$. Anal. calcd for $\mathrm{C}_{41} \mathrm{H}_{38} \mathrm{~N}_{4} \mathrm{O}_{4} \mathrm{C} 75.67, \mathrm{H} \mathrm{5.89,} \mathrm{N}$ 8.61; found C 75.56, H 6.00, N 8.81;

\subsection{2(E)-苯亚甲基红紫素-18 甲酯(11)的合成}

将 $117 \mathrm{mg}$ 化合物 $\mathbf{1 0}(0.180 \mathrm{mmol})$ 溶解于 $10 \mathrm{~mL}$ 甲 醇中, 再加入 $4 \mathrm{~mL}$ 三乙胺和 $80 \mathrm{mg}$ 研细的 $\mathrm{KOH}$ 粉末, $35{ }^{\circ} \mathrm{C}$ 开口搅拌 $5 \mathrm{~h}$, 减压除尽溶剂, 将剩余物再溶解于 $10 \mathrm{~mL}$ 乙酸中, 室温搅拌 $6 \mathrm{~h}$, 反应结束后倒入 $150 \mathrm{~g}$ 碎 冰中, 然后加入 $20 \mathrm{~mL}$ 二氯甲烷, 水层用二氯甲烷萃取 $(20 \mathrm{~mL} \times 3)$, 合并有机层, 水洗两次, 用无水硫酸钠干 燥, 减压除去溶剂后重新溶解于 $5 \mathrm{~mL}$ 二氯甲烷中, 加 入过量的重氮甲烷甲基化, 震摇 $3 \mathrm{~min}$ 后用乙酸淬灭反 应, 浓缩, 剩余物用硅胶柱层析[洗脱剂: $V$ 甲羑 $: V$ 两醂 $=$ $10: 1]$ 分离反应混合物, 得 $55 \mathrm{mg}$ 红色固体 $11(0.083$ mmol), 产率 46\%. m.p. 202 205 ${ }^{\circ} \mathrm{C}$; UV-vis $\left(\mathrm{CH}_{2} \mathrm{Cl}_{2}\right)$

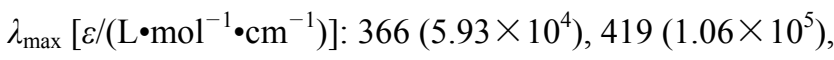
$524\left(9.54 \times 10^{3}\right), 561\left(1.48 \times 10^{4}\right), 724\left(4.24 \times 10^{4}\right) \mathrm{nm} ;{ }^{1} \mathrm{H}$ NMR $\left(\mathrm{CDCl}_{3}\right) \delta: 0.24$ (br s, $\left.1 \mathrm{H}, \mathrm{NH}\right), 0.35$ (br s, 1H, NH), 1.50 (t, $\left.J=7.6 \mathrm{~Hz}, 3 \mathrm{H}, 8-\mathrm{CH}_{3}\right), 1.76(\mathrm{~d}, J=6.8 \mathrm{~Hz}, 3 \mathrm{H}$, $\left.18-\mathrm{CH}_{3}\right), 1.94 \sim 2.02(\mathrm{~m}, 1 \mathrm{H}, 17 \mathrm{a}+17 \mathrm{~b}-\mathrm{H}), 2.47$ (d, $J=$ $12.2 \mathrm{~Hz}, 1 \mathrm{H}, 17 \mathrm{a}+17 \mathrm{~b}-\mathrm{H}), 2.53(\mathrm{~d}, J=10.2 \mathrm{~Hz}, 1 \mathrm{H}$, $17 \mathrm{a}+17 \mathrm{~b}-\mathrm{H}), \quad 2.74 \sim 2.85(\mathrm{~m}, 1 \mathrm{H}, 17 \mathrm{a}+17 \mathrm{~b}-\mathrm{H}), 3.05$, 3.24, 3.63 (each s, each 3H, $\mathrm{CH}_{3}+\mathrm{OCH}_{3}$ ), 3.35 (q, $J=7.6$ $\mathrm{Hz}, 2 \mathrm{H}, 8 \mathrm{a}-\mathrm{H}), 4.34$ (q, $J=7.6 \mathrm{~Hz}, 1 \mathrm{H}, 18-\mathrm{H}), 5.17$ (d, $J=$ $9.2 \mathrm{~Hz}, 1 \mathrm{H}, 17-\mathrm{H}), 6.15$ (d, J=11.2 Hz, cis-3b-H), 6.26 (d, $J=17.6 \mathrm{~Hz}$, trans-3b-H), 7.45 (t, $J=7.0 \mathrm{~Hz}, 1 \mathrm{H}, \mathrm{ArH})$, 
$7.53(\mathrm{t}, J=7.0 \mathrm{~Hz}, 2 \mathrm{H}, \mathrm{ArH}), 7.65 \sim 7.73(\mathrm{~m}, 3 \mathrm{H}, 12 \mathrm{~b}-\mathrm{H}+$ ArH), 7.80 (dd, $J=17.6,11.2 \mathrm{~Hz}, 1 \mathrm{H}, 3 \mathrm{a}-\mathrm{H}), 8.26$ (dd, $J=$ 16.0, $1.0 \mathrm{~Hz}, 12 \mathrm{a}-\mathrm{H}$ ), 8.44, 9.15, 9.16 (each s, each $1 \mathrm{H}$, meso-H); IR (KBr) v: $3450(\mathrm{~N}-\mathrm{H}), 2925,2860(\mathrm{C}-\mathrm{H})$, 1743, $1708(\mathrm{C}=\mathrm{O}), 1604(\mathrm{C}=\mathrm{C}), 1531$ (chlorin skeleton), $1400,1311,1134,902,707 \mathrm{~cm}^{-1}$. Anal. calcd for $\mathrm{C}_{41} \mathrm{H}_{38^{-}}$ $\mathrm{N}_{4} \mathrm{O}_{5} \mathrm{C} 73.85, \mathrm{H} 5.74, \mathrm{~N} 8.40$; found $\mathrm{C} 73.98, \mathrm{H} 5.60, \mathrm{~N}$ 8.59 .

\subsection{2(E)-12-苯亚甲基二氢卟吩- $\mathrm{e}^{6}$ 三甲酯(12)的合成}

将 $128 \mathrm{mg}$ 二氢卟吩二酮 $\mathbf{1 0}(0.197 \mathrm{mmol})$ 溶解于含 有 $15 \mathrm{mg}$ 金属钠的甲醇溶液中, 室温剧烈搅拌反应 $6 \mathrm{~h}$; 加入 $25 \mathrm{~mL}$ 二氯甲烷和 $40 \mathrm{~mL}$ 水分层, 分出有机相并用 水洗 $(20 \mathrm{~mL} \times 3)$, 将反应液浓缩至 $3 \mathrm{~mL}$, 加入过量的重 氮甲烷甲基化, 震摇 $3 \mathrm{~min}$ 后用乙酸淬灭反应, 浓缩, 剩 余物硅胶柱层析分离 [洗脱剂: $V_{\text {乙酸乙酯 }}: V_{\text {正已烷 }}=4: 1$ ] 得 到 $82 \mathrm{mg}$ 红色固体 12 (0.114 mmol), 产率 58\%. m.p.

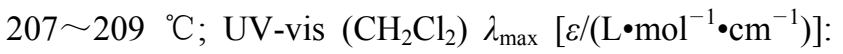
$411\left(9.79 \times 10^{4}\right), 507\left(8.81 \times 10^{3}\right), 539\left(6.85 \times 10^{3}\right), 627$ $\left(7.83 \times 10^{3}\right), 681\left(3.82 \times 10^{4}\right) \mathrm{nm} ;{ }^{1} \mathrm{H}$ NMR $(400 \mathrm{~Hz}$, $\left.\mathrm{CDCl}_{3}\right) \delta:-0.37$ (br s, $\left.1 \mathrm{H}, \mathrm{NH}\right),-0.33$ (br s, $1 \mathrm{H}, \mathrm{NH}$ ), $1.62\left(\mathrm{t}, J=7.5 \mathrm{~Hz}, 3 \mathrm{H}, 18-\mathrm{CH}_{3}\right), 1.86(\mathrm{~d}, J=7.2 \mathrm{~Hz}, 3 \mathrm{H}$, $\left.8-\mathrm{CH}_{3}\right), 1.78 \sim 1.90(\mathrm{~m}, 1 \mathrm{H}, 17 \mathrm{a}+17 \mathrm{~b}-\mathrm{H}), 2.05 \sim 2.24(\mathrm{~m}$, $2 \mathrm{H}, 17 \mathrm{a}+17 \mathrm{~b}-\mathrm{H}), 2.32 \sim 2.40(\mathrm{~m}, 1 \mathrm{H}, 17 \mathrm{a}+17 \mathrm{~b}-\mathrm{H}), 3.13$, $3.32,3.53,4.16,4.21$ (each s, each $3 \mathrm{H}, \mathrm{CH}_{3}+\mathrm{OCH}_{3}$ ), 3.55 (q, $J=7.5 \mathrm{~Hz}, 2 \mathrm{H}, 8 \mathrm{a}-\mathrm{H}), 4.34$ (q, $J=7.2 \mathrm{~Hz}, 1 \mathrm{H}, 18-\mathrm{H})$, 5.17 (d, 1H, $J=8.4 \mathrm{~Hz}, 1 \mathrm{H}, 17-\mathrm{H}), 6.07$ (d, $J=10.8 \mathrm{~Hz}$, $1 \mathrm{H}$, cis-3b-H), 6.24 (d, J=17.6 Hz, 1H, trans-3b-H), 7.36 $(\mathrm{d}, J=16.4 \mathrm{~Hz}, 1 \mathrm{H}, 12 \mathrm{~b}-\mathrm{H}), 7.42 \sim 7.53$ (m, 3H, ArH), $7.81 \sim 7.88(\mathrm{~m}, 3 \mathrm{H}, 3 \mathrm{a}-\mathrm{H}+\mathrm{ArH}), 8.64(\mathrm{~d}, J=16.4 \mathrm{~Hz}, 1 \mathrm{H}$, 12a-H), 8.53, 9.26, 9.77 (each s, each 1H, meso-H); IR $(\mathrm{KBr}) v: 3324(\mathrm{~N}-\mathrm{H}), 2925(\mathrm{C}-\mathrm{H}), 1739,1719(\mathrm{C}=\mathrm{O})$, $1602(\mathrm{C}=\mathrm{C}), 1500$ (chlorin skeleton), 1451, 1263, 1089, 1041, 902, $700 \mathrm{~cm}^{-1}$. Anal. calcd for $\mathrm{C}_{43} \mathrm{H}_{44} \mathrm{~N}_{4} \mathrm{O}_{6} \mathrm{C} 72.45$, H 6.22, N 7.86; found C 72.60, H 6.31, N 7.90.

\subsection{2(E)- $N$-甲氧基-12-苯亚甲基红紫素-18 二酰亚胺 甲酯(13)的合成}

将 $105 \mathrm{mg}$ 红紫素-18 甲酯 11 (0.157 mmol)溶解于 $15 \mathrm{~mL}$ 吡啶中, 并向其中加入过量的盐酸羟胺 (390 mg), 室温下摚拌反应 $5 \mathrm{~h}$, 先后向反应体系加入 $6 \mathrm{~mL}$ 浓盐酸 和 $200 \mathrm{~mL}$ 水, 然后用二氯甲烷萃取 $(100 \mathrm{~mL} \times 3)$, 合并 有机相并水洗除酸, 减压除尽溶剂后重新溶解在 $10 \mathrm{~mL}$ 二氯甲烷中, 加入过量的自制重氮甲烷乙醚溶液, 振摇 $3 \mathrm{~min}$, 减压蒸除溶剂, 剩余物经硅胶柱层析[展开剂: $\left.V_{\text {甲苯 }}: V_{\text {丙醂 }}=20: 1\right]$, 得 $83 \mathrm{mg}$ 红色产物 13 (0.120 mmol) 产率为 $76 \%$. m.p. $215 \sim 218{ }^{\circ} \mathrm{C}$; UV-vis $\left(\mathrm{CHCl}_{3}\right) \lambda_{\max }$

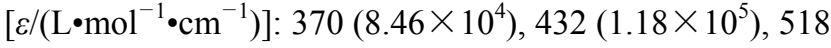
$\left(5.91 \times 10^{3}\right), \quad 564 \quad\left(3.06 \times 10^{4}\right), \quad 657 \quad\left(8.26 \times 10^{3}\right), \quad 730$ $\left(6.61 \times 10^{4}\right) \mathrm{nm} ;{ }^{1} \mathrm{H}$ NMR $\left(\mathrm{CDCl}_{3}\right) \delta: 0.47$ (br s, $\left.1 \mathrm{H}, \mathrm{NH}\right)$, 0.53 (br s, 1H, NH), 1.55 (t, $J=7.6 \mathrm{~Hz}, 3 \mathrm{H}, 8-\mathrm{CH}_{3}$ ), 1.73 $\left(\mathrm{d}, J=7.3 \mathrm{~Hz}, 3 \mathrm{H}, 18-\mathrm{CH}_{3}\right), 1.90 \sim 2.01(\mathrm{~m}, 1 \mathrm{H}, 17 \mathrm{a}+$ $17 \mathrm{~b}-\mathrm{H}), 2.43 \sim 2.58(\mathrm{~m}, 2 \mathrm{H}, 17 \mathrm{a}+17 \mathrm{~b}-\mathrm{H}), 2.80 \sim 2.87(\mathrm{~m}$, $1 \mathrm{H}, 17 \mathrm{a}+17 \mathrm{~b}-\mathrm{H}$ ), 3.02, 3.26, 3.60, 4.36 (each s, each $3 \mathrm{H}$, $\mathrm{CH}_{3}+\mathrm{OCH}_{3}$ ), 3.38 (q, $\left.J=7.6 \mathrm{~Hz}, 2 \mathrm{H}, 8 \mathrm{a}-\mathrm{H}\right), 4.31$ (q, $J=$ $7.4 \mathrm{~Hz}, 1 \mathrm{H}, 18-\mathrm{H}), 5.25$ (dd, $J=9.6,2.2 \mathrm{~Hz}, 1 \mathrm{H}, 17-\mathrm{H})$, $6.12(\mathrm{~d}, J=11.5 \mathrm{~Hz}$, cis-3b-H), 6.22 (d, $J=17.5 \mathrm{~Hz}$, trans-3b-H), 7.44 (t, $J=7.3 \mathrm{~Hz}, 1 \mathrm{H}, \mathrm{ArH}), 7.53$ (t, $J=7.3$ $\mathrm{Hz}, 2 \mathrm{H}, \mathrm{ArH}), 7.78$ (dd, $J=17.8,11.5 \mathrm{~Hz}, 1 \mathrm{H}, 3 \mathrm{a}-\mathrm{H}), 7.72$ (d, $J=16.5 \mathrm{~Hz}, 12 \mathrm{~b}-\mathrm{H}), 7.81$ (t, $J=7.3 \mathrm{~Hz}, 2 \mathrm{H}, \mathrm{ArH}), 8.68$ (d, $J=16.5 \mathrm{~Hz}, 1 \mathrm{H}, 12 \mathrm{a}-\mathrm{H}), 9.42,9.32,8.42$ (each s, each 1H, meso-H); IR (KBr) v: $3458(\mathrm{O}-\mathrm{H}), 2856(\mathrm{C}-\mathrm{H})$, 1737, $1704(\mathrm{C}=\mathrm{O}), 1616(\mathrm{C}=\mathrm{C}), 1571$ (chlorin skeleton), 1481, 1438, 1265, 1081, $1022 \mathrm{~cm}^{-1}$; EI-MS m/z: 696.2 $\left(\mathrm{MH}^{+}\right)$. Anal. calcd for $\mathrm{C}_{41} \mathrm{H}_{42} \mathrm{~N}_{5} \mathrm{O}_{5} \mathrm{C}$ 72.50, H 5.94, N 10.07; found C 72.39, H 5.88, N 9.98.

$3.1012(E)-12$-苯亚甲基-(2,3-n)-喹喔啉并焦脱镁叶绿 酸- $a$ 甲酯(14)和 12(E)-12-苯亚甲基-(2,3-n)-苯并咪唑并 红紫素-18 甲酯(15)的合成

将 $127 \mathrm{mg}$ 化合物 $\mathbf{1 0}(0.195 \mathrm{mmol})$ 溶解于 $20 \mathrm{~mL}$ 吡 啶中, 加入 $0.05 \mathrm{~mL}$ 三氟乙酸、 $2 \mathrm{~mL}$ 乙酸和 $60 \mathrm{mg}$ 邻苯 二胺 $(0.555 \mathrm{mmol})$, 室温搅拌 $6 \mathrm{~h}$, 减压除尽吡啶, 向所 得到浓缩物加水和二氯甲烷分层, 用二氯甲烷萃取水 相，合并有机层并水洗，干燥后减压浓缩，硅胶柱层析 分离[展开剂: $V_{\text {石油醚 }}: V_{\text {乙酸乙酯 }}=3: 1$ ], 得到 $25 \mathrm{mg}$ 黄色固 体 14 (0.035 mmol, 18\%)和 $23 \mathrm{mg}$ 橙色固体 15 (0.031 mmol, 16\%).

14: m.p. $208 \sim 211{ }^{\circ} \mathrm{C}$; UV-vis $\left(\mathrm{CHCl}_{3}\right) \lambda_{\max }$

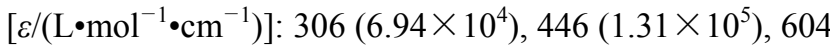
$\left(1.18 \times 10^{4}\right), 682\left(2.10 \times 10^{4}\right), 738\left(6.71 \times 10^{4}\right) \mathrm{nm} ;{ }^{1} \mathrm{H}$ NMR $\left(\mathrm{CDCl}_{3}\right) \delta$ : -0.69 (br s, $\left.1 \mathrm{H}, \mathrm{NH}\right), 0.45$ (br s, 1H, $\mathrm{NH}), 1.54$ (t, $\left.J=7.6 \mathrm{~Hz}, 3 \mathrm{H}, 8-\mathrm{CH}_{3}\right), 1.96$ (d, $J=6.8 \mathrm{~Hz}$, $\left.3 \mathrm{H}, 18-\mathrm{CH}_{3}\right), 2.25 \sim 2.35(\mathrm{~m}, 2 \mathrm{H}, 17 \mathrm{a}+17 \mathrm{~b}-\mathrm{H}), 2.44 \sim$ $2.55(\mathrm{~m}, 1 \mathrm{H}, 17 \mathrm{a}+17 \mathrm{~b}-\mathrm{H}), 2.72 \sim 2.83(\mathrm{~m}, 1 \mathrm{H}, 17 \mathrm{a}+$ 17b-H), 2.89, 3.19, 3.61 (each s, each $3 \mathrm{H}, \mathrm{CH}_{3}+\mathrm{OCH}_{3}$ ), 3.39 (q, $J=7.6 \mathrm{~Hz}, 2 \mathrm{H}, 8 \mathrm{a}-\mathrm{H}), 4.41$ (q, $J=7.2 \mathrm{~Hz}, 1 \mathrm{H}$, $18-\mathrm{H}), 5.04$ (d, $J=8.4 \mathrm{~Hz}, 1 \mathrm{H}, 17-\mathrm{H}), 6.11$ (d, $J=11.6 \mathrm{~Hz}$, cis-3b-H), $6.21(\mathrm{~d}, J=18.0 \mathrm{~Hz}$, trans-3b-H), $7.38 \sim 7.42$ (m, 1H, ArH), $7.45 \sim 7.55$ (m, 4H, ArH), 7.69 (d, $J=15.4$ $\mathrm{Hz}, 1 \mathrm{H}, 12 \mathrm{~b}-\mathrm{H}), 7.66 \sim 7.81$ (m, 3H, 12b-H+ArH), 7.85 (dd, $J=18.0,11.6 \mathrm{~Hz}, 1 \mathrm{H}, 3 \mathrm{a}-\mathrm{H}), 7.95$ (d, $J=7.2 \mathrm{~Hz}, 1 \mathrm{H}$, ArH), 8.73 (d, $J=15.4 \mathrm{~Hz}, 1 \mathrm{H}, 12 \mathrm{a}-\mathrm{H}), 8.29,8.83,9.10$ 
(each s, each $1 \mathrm{H}$, meso-H); IR $(\mathrm{KBr})$ v: $3458(\mathrm{~N}-\mathrm{H})$, 2918, $2850(\mathrm{C}-\mathrm{H}), 1733,1704(\mathrm{C}=\mathrm{O}), 1623(\mathrm{C}=\mathrm{C})$, 1558 (chlorin skeleton), 1465, 1170. 1041, 918, $736 \mathrm{~cm}^{-1}$. Anal. calcd for $\mathrm{C}_{47} \mathrm{H}_{42} \mathrm{~N}_{6} \mathrm{O}_{2} \mathrm{C}$ 78.09, H 5.86, N 11.63; found $\mathrm{C} 78.17, \mathrm{H} 5.77, \mathrm{~N} 11.88$.

15: m.p. $216 \sim 219{ }^{\circ} \mathrm{C}$; UV-vis $\left(\mathrm{CHCl}_{3}\right) \lambda_{\max }$

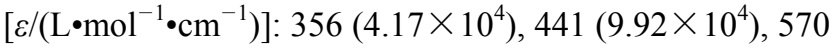
$\left(1.98 \times 10^{4}\right), 687\left(1.19 \times 10^{4}\right), 750\left(4.76 \times 10^{4}\right) \mathrm{nm} ;{ }^{1} \mathrm{H}$ NMR $\left(\mathrm{CDCl}_{3}\right) \delta: 0.27$ (br s, $\left.1 \mathrm{H}, \mathrm{NH}\right), 0.42($ br s, $1 \mathrm{H}, \mathrm{NH})$, $1.57\left(\mathrm{t}, J=7.6 \mathrm{~Hz}, 3 \mathrm{H}, 8-\mathrm{CH}_{3}\right), 1.80(\mathrm{~d}, J=7.2 \mathrm{~Hz}, 3 \mathrm{H}$, $\left.18-\mathrm{CH}_{3}\right), 2.17 \sim 2.35(\mathrm{~m}, 1 \mathrm{H}, 17 \mathrm{a}+17 \mathrm{~b}-\mathrm{H}), 2.40 \sim 2.54$ (m, 2H, 17a+17b-H), 2.73 2.85 (m, 1H, 17a+17b-H), 2.97, 3.27, 3.61 (each s, each $3 \mathrm{H}, \mathrm{CH}_{3}+\mathrm{OCH}_{3}$ ), 3.41 (q, $J=7.6 \mathrm{~Hz}, 2 \mathrm{H}, 8 \mathrm{a}-\mathrm{H}), 4.32$ (q, $J=7.2 \mathrm{~Hz}, 1 \mathrm{H}, 18-\mathrm{H}), 5.41$ (d, $J=8.8 \mathrm{~Hz}, 1 \mathrm{H}, 17-\mathrm{H}), 6.13$ (d, $J=11.2 \mathrm{~Hz}$, cis-3b-H), $6.27(\mathrm{~d}, J=17.2 \mathrm{~Hz}$, trans-3b-H), $7.43 \sim 7.60(\mathrm{~m}, 3 \mathrm{H}$, ArH), $7.74 \sim 7.95$ (m, 5H, 3a-H+ArH), 8.03 (d, $J=15.2$ $\mathrm{Hz}, 1 \mathrm{H}, 12 \mathrm{~b}-\mathrm{H}), 8.13 \sim 8.22$ (m, 2H, ArH), 8.63 (d, $J=$ $15.2 \mathrm{~Hz}, 1 \mathrm{H}, 12 \mathrm{a}-\mathrm{H}), 8.72 \sim 8.76$ (m, 1H, ArH), 8.46, 9.16, 9.44 (each s, each 1H, meso-H); IR (KBr) v: $3448 \quad(\mathrm{~N}-$ H), $2926(\mathrm{C}-\mathrm{H})$, 1739, $1690(\mathrm{C}=\mathrm{O}), 1655(\mathrm{C}=\mathrm{C}), 1551$ (chlorin skeleton), 1080, $780 \mathrm{~cm}^{-1}$. Anal. calcd for $\mathrm{C}_{47} \mathrm{H}_{42} \mathrm{~N}_{6} \mathrm{O}_{3}$ C 76.40, H 5.73, N 11.37; found C 76.27, H 5.77, N 11.28 .

辅助材料(Supporting Information) 化合物 $2 \sim 15$ 的 ${ }^{1} \mathrm{H}$ NMR 谱数据. 这些材料可以免费从本刊网站 (http://sioc-journal.cn/)上下载.

\section{References}

[1] (a) Pavlov, V. Y.; Ponomarev, G. V. Chem. Heterocycl. Compd. 2004, 40, 393.

(b) Chen, Y. H.; Li, G. L.; Pandey, R. K. Cur. Org. Chem. 2004, 8, 1105 .

(c) Wang, J.-J. Chin. J. Org. Chem. 2005, 25, 1353 (in Chinese). (王进军, 有机化学, 2005, 25, 1353.)

(d) Yang, Z.; Wang, Z.; Liu, Y.; Xu, X.-S.; Qi, C.-X.; Wang, J.-J. Chin. J. Org. Chem. 2013, 33, 116 (in Chinese).

(杨泽, 王振, 刘洋, 徐希森, 祁彩霞, 王进军, 有机化学, 2013,
$33,116$.

[2] (a) Kozyrev, A.; Ethirajan, M,; Chen, P.; Ohkubo, K.; Robinson, GB. C.; Berkigia, K. M.; Fukuzumi, S.; Kadish, K. M.; Pandey, J. J. Org. Chem. 2012, 77, 10260.

(b) Kozyrey, A. N.; Chen, Y.-H.; Goswami, L. N.; Tabaczynaki, W. A.; Pandey, R. K. J. Org. Chem. 2006, 71, 1949.

(c) Liu, R.-R.; Wang, L.-M.; Yin, J.-G.; Wu, J.; Liu, C.; Zhang, P.; Wang, J.-J. Chin. J. Org. Chem. 2012, 32, 318 (in Chinese).

(刘舟舟, 王鲁敏, 金英学, 武进, 刘超, 王朋, 王进军, 有机化 学, 2012, 32, 318.)

[3] (a) Wang, J.-J.; Yin, Y.-F.; Yang, Z. J. Iran. Chem. Soc. 2013, 10, 583.

(b) Tamiaki, H.; Monobe, R.; Koizumi, S.; Miyatake, T.; Kinoshita, Y. Tetrahedron: Asymmerty 2013, 24, 966.

(c) Srivatsan, A.; Wang, Y.-F.; Joshi, P.; Sajjad, M.; Chen, Y.-H.; Liu, C.; Thankcppan, K.; Missert, J. R.; Tracy, E.; Morgan, J.; Rigual, N.; Baumann, H.; Pandey, R. K. J. Med. Chem. 2011, 54, 6859.

[4] (a) Li, J.-Z.; Liu, W.-H.; Li, F.-G.; Wang, J.-J.; Suo, Y.-R.; Liu, Y.-J. Chin. J. Org. Chem. 2007, 27, 1594 (in Chinese).

(李家柱, 刘万卉, 李付国, 王进军, 索有瑞, 刘永军, 有机化学, 2007, 27, 1594.)

(b) Wang, J.-J.; Li, J.-Z.; Jakus, J.; Shin, K. J. Porphyrins Phthalocyanines 2012, 16, 122.

[5] (a) Li, J.-Z.; Wang, J.-J.; Yoon, L.; Cui, B.-C.; Shim, Y.-K. Bioorg. Med. Chem. Lett. 2012, 22, 1846.

(b) Wang, L.-M.; Wang, P.; Liu, C.; Jin, Y.-X.; Wang, J.-J. Chin. J. Org. Chem. 2012, 32, 1707 (in Chinese).

(王鲁敏, 王朋, 刘超, 金英学, 王进军, 有机化学, 2012, 32, 1707.)

[6] Ji, J.-Y.; Wang, L. M.; Jing, J.-R.; Han, G.-F.; Wang, J.-J. Chin. J. Org. Chem. 2007, 27, 493 (in Chinese).

(纪建业, 王鲁民, 荆济荣, 韩光范, 王进军，有机化学，2007, 27, 493.)

[7] Li, Y.-W. M.S. Thesis, Yantai University, Yantai, 2009 (in Chinese). (李韵伟, 硕士论文, 烟台大学, 烟台, 2009.)

[8] (a) Yin, J.-G.; Wang, Z.; Yang, Z.; Liu, C.; Zhao, L.-L.; Wang, J.-J. Chin. J. Org. Chem. 2012, 32, 360 (in Chinese). (殷军港, 王振, 杨泽, 刘超, 赵丽丽, 王进军, 有机化学, 2012, 32,360 .)

(b) Wang, J.-J.; Zhao, Y.; Wu, X.-R.; Han, G.-F.; Shin, R.-K. Chin. J. Org. Chem. 2002, 22, 565 (in Chinese).

(王进军, 赵岩, 邬旭然, 韩光范. 沈荣基, 有机化学, 2002, 22, 565.)

(c) Shin-ichi Sasaki, S.; Yoshizato, M.; Kunieda, M.; Tamiaki, H. Eur. J. Org. Chem. 2010, 5287.

[9] Wu, J. M.S. Thesis, Yantai University, Yantai, 2011 (in Chinese). (武进, 硕士论文, 烟台大学, 烟台, 2011.)

[10] Smith, K. M.; Gogg, D. A.; Simpson, D. J. J. Am. Chem. Soc. 1985, $107,4946$. 